\title{
Sutured Scleral Fixation of Posterior Chamber Intraocular Lens in Children under the Age of 9 with Congenital Ectopia Lentis
}

\author{
Xuyun Meng ${ }^{\mathrm{a}} \quad \mathrm{Xi} \mathrm{Cao}^{\mathrm{a}}$ Yiyue Jia $^{\mathrm{a}}$ Jianhui Pan ${ }^{\mathrm{b}} \quad \mathrm{Yi} \mathrm{Du}^{\mathrm{a}} \quad \mathrm{Xia} \mathrm{Li}^{\mathrm{a}}$ \\ aDepartment of Ophthalmology, The First Affiliated Hospital of Guangxi Medical University, Nanning, China; \\ ${ }^{b}$ Department of Ophthalmology, Guiping City People's Hospital, Guiping, China
}

\section{Keywords}

Scleral fixation · Implants · Congenital ectopia lentis ·

Children $\cdot$ Intraocular lens

\begin{abstract}
Introduction: This study aimed to report the visual acuity outcomes and complications of sutured scleral fixation of posterior chamber intraocular lens (IOL) in children under 9 years old with congenital ectopia lentis. Methods: Twentysix children (47 eyes) with congenital ectopia lentis were included in this study. The mean age at surgery was $61.6 \pm 22.3$ months (range, 32-94). Patients underwent lens extraction, anterior vitrectomy, and sutured scleral fixation of posterior chamber IOL. The implanted IOL included rigid polymethyl methacrylate (PMMA) IOL (CZ70BD, $n=36)$ and foldable IOL (SA60AT, $n=9$, and LI61SE, $n=2$ ). The outcome measures used to assess the benefits were uncorrected visual acuity (UCVA), best-corrected visual acuity (BCVA), refraction, intraocular pressure, and any associated complications. Median follow-up was 33.0 months (range, 4-129). Results: After surgery, the median UCVA (1.30 vs. 0.35 logMAR) and BCVA (0.82 vs. 0.15 logMAR) improved significantly $(p<0.001)$. The median absolute spherical values decreased considerably ( 9.00 vs. $0.75 \mathrm{D} ; p<0.001)$. The median astigmatism was lower in foldable IOL compared to rigid PMMA IOL (1.0 vs. 2.5 D; $p=0.026)$, but neither the UCVA nor BCVA was significantly
\end{abstract}

different. There was no intraoperative complication. Postoperative complications included pupillary capture in 4 eyes (9\%), IOL decentration in 4 eyes (9\%), choroid edema in 1 eye (2\%), and subretinal hemorrhage in 1 eye (2\%). The rate of secondary surgery was $9 \%$, caused by IOL decentration of IOL haptics which was broken in 3 eyes and suture degradation in 1 eye. Conclusion: Sutured scleral fixation of posterior chamber IOL provided good visual outcomes in children under 9 years of age with congenital ectopia lentis. Although there were some risks of secondary surgery, the complications were acceptable.

(c) 2021 The Author(s)

Published by S. Karger AG, Basel

\section{Introduction}

Congenital ectopia lentis is an uncommon ocular disease presenting with subluxated or dislocated lentis due to the hypoplasia of the zonular. It had a prevalence rate of approximately 6.4/100,000 in Denmark in 1993 [1]. It can be idiopathic or associated with an inherited connective tissue disorder such as Marfan syndrome, homocystinuria, Weill-Marchesani syndrome, and Ehlers-Danlos syndrome. Children with severe ectopia lentis can be observed at a young age, which can result in high amblyopia and poor visual acuity, and surgical intervention is often necessary.

Correspondence to:

Xia Li, lixiagmu066@163.com

karger@karger.com www.karger.com/ore

Karger $\stackrel{\text { ' }}{=}$
(C) 2021 The Author(s)

Published by S. Karger AG, Basel

This is an Open Access article licensed under the Creative Commons Attribution-NonCommercial-4.0 International License (CC BY-NC) (http://www.karger.com/Services/OpenAccessLicense), applicable to the online version of the article only. Usage and distribution for commercial purposes requires written permission. 
Table 1. Visual and refractive preoperative and postoperative outcomes

\begin{tabular}{lllr}
\hline & $\begin{array}{l}\text { Preoperative median } \\
\text { (range) }\end{array}$ & $\begin{array}{l}\text { Postoperative median } \\
\text { (range) }\end{array}$ & $p$ value $^{\mathrm{a}}$ \\
\hline UCVA, logMAR & $1.30(0.30-2.00)$ & $0.35(0.15-1.00)$ & $<0.001$ \\
BCVA, logMAR & $0.82(0.30-1.30)$ & $0.15(0.00-0.82)$ & $<0.001$ \\
Spherical, D & $9.50(2.50-18.00)$ & $0.75(0.00-3.25)$ & $<0.001$ \\
Astigmatism, D & $1.50(0.00-7.00)$ & $2.25(0.00-4.50)$ & 0.126 \\
Spherical equivalent, D & $4.00(-17.00$ to 18.00$)$ & $1.38(-2.50$ to 4.38$)$ & 0.638 \\
\hline
\end{tabular}

UCVA, uncorrected visual acuity; BCVA, best-corrected visual acuity. ${ }^{\text {a }}$ Wilcoxon signed-rank test. ${ }^{\mathrm{b}}$ The astigmatism of diopter.
Table 2. A comparison of either PMMA or foldable IOL procedures

\begin{tabular}{llll}
\hline & $\begin{array}{l}\text { PMMA IOL median } \\
\text { (range) }\end{array}$ & $\begin{array}{l}\text { Foldable IOL median } \\
\text { (range) }\end{array}$ & $p$ value $^{\mathrm{a}}$ \\
\hline UCVA, logMAR & $0.40(0.22-0.80)$ & $0.30(0.15-1.25)$ & 0.756 \\
BCVA, logMAR & $0.19(0.05-0.82)$ & $0.15(0.00-0.50)$ & 0.101 \\
Astigmatism, D & $2.50(0.50-4.50)$ & $1.00(0.00-4.25)$ & 0.026 \\
Spherical equivalent, D & $1.57(-2.25$ to 4.38$)$ & $0.25(-2.50$ to 2.25$)$ & 0.086 \\
\hline
\end{tabular}

IOL, intraocular lens; PMMA, polymethyl methacrylate; UCVA, uncorrected visual acuity; BCVA, best-corrected visual acuity. ${ }^{\mathrm{a}}$ Mann-Whitney $\mathrm{U}$ test. ${ }^{\mathrm{b}}$ The astigmatism of diopter.
Various methods of lens implantation have been employed in children with ectopia lentis after lens removal, but the best option remains uncertain. Anterior chamber intraocular lens (IOL) and iris fixated IOL should be placed with caution as these are often associated with the postoperative complications such as corneal endothelial damage, glaucoma, and IOL dislocation [2-7]. In some cases, IOL and traditional capsular tension ring implantation can lead to further decentration or even dislocation requiring further surgical revisions [8]. However, a modified capsular tension ring can be prohibited by the extensive degree of zonulopathy or small size of the capsular bag [9].

Sutured scleral fixation of posterior chamber IOL (SFPCIOL) is a feasible and relatively widespread technology. Some studies had reported it is an effective and safe way to proceed for children with inadequate capsular support [10-12]. However, in younger patients with congenital ectopia lentis, the probability of complications, such as pupillary capture, is still high [13]. In addition, in these patients, the surgery required is more complicated and is often associated with the hypoplastic nature of the ocular tissue, peculiarities in the anatomy of the ocular tissue, and a risk of severe postoperative inflammation [14]. There are only limited data on the surgical outcomes of sutured SF-PCIOL in the dislocated lens of young chil-
Table 3. Postoperative complications in eyes after SF-PCIOL

\begin{tabular}{ll}
\hline Complications & Eyes, $n(\%)$ \\
\hline Pupillary capture & $4(9)$ \\
IOL decentration & $4(9)$ \\
Choroid edema & $1(9)$ \\
Subretinal hemorrhage & $1(2)$ \\
\hline
\end{tabular}

IOL, intraocular lens; SF-PCIOL, scleral fixation of posterior chamber IOL.

dren with congenital ectopia lentis. Herein, we performed a retrospective study to evaluate the visual outcomes and possible complications after sutured SF-PCIOL in 26 children (encompassing 47 eyes) under 9 years of age with congenital ectopia lentis.

\section{Materials and Methods}

We reviewed the clinical records of 26 children (47 eyes) with congenital ectopia lentis, retrospectively. The average age of the patients was $61.6 \pm 22.3$ months, and they underwent lens extraction, anterior vitrectomy, and SF-PCIOL by 3 experienced doctors, at the First Affiliated Hospital of Guangxi Medical University, Chi- 
Table 4. Details of patients who underwent secondary surgery

\begin{tabular}{lllllll}
\hline Case & Age & Eye & $\begin{array}{l}\text { Post- } \\
\text { operative }\end{array}$ & Complications & Secondary surgery & BCVA* $^{*}$ \\
\hline 1 & 6 yr 8 mo & Left & $1 \mathrm{~d}$ & IOL haptics broken & IOL replacement and IOL SF-PCIOL & 0.3 \\
2 & 3 yr 7 mo & Right & 72 mo & IOL haptics broken & IOL replacement and IOL SF-PCIOL & 1.0 \\
& & Left & 72 mo & IOL haptics broken & IOL replacement and IOL SF-PCIOL & 0.9 \\
3 & 3 yr 10 mo & Right & 7 mo & Suture degradation & IOL re-fixation & 0.7 \\
\hline
\end{tabular}

IOL, intraocular lens; SF-PCIOL, scleral fixation of posterior chamber IOL; BCVA, best-corrected visual acuity. ${ }^{*}$ BCVA at the final visit.

na, from May 5, 2010, to October 31, 2020. This study was performed in line with the principles of the Declaration of Helsinki. Approval was granted by the Ethics Committee of the First Affiliated Hospital of Guangxi Medical University (No. 2020.KY-E-171). All data were anonymized and collected retrospectively, and the requirement for written informed consent was therefore waived.

Preoperatively, the data collected included age, sex, uncorrected visual acuity (UCVA, $\log M A R)$, best-corrected visual acuity (BCVA, logMAR), refraction, intraocular pressure (IOP), and axial length. IOP was obtained using a noncontact tonometer (CT800; Topcon, Tokyo, Japan) or a Schiotz tonometer (YZ7A; 66 Vision Tech Co. Ltd., Suzhou, China) under phenobarbital anesthesia, and axial length was measured by using A-scan ultrasound (AVISO; Quantel Medical, Cournon-d'Auvergne, France). In some patients, both A-scan ultrasound and IOL-master biometer (Carl Zeiss Meditec AG, Jena, Germany) were used. The anterior segment and degree of crystalline lens dislocation were examined using a slit-lamp. The posterior segment was examined by using B-ultrasound (AVISO; Quantel Medical, Cournon-d'Auvergne, France) as well as by direct ophthalmoscopy.

All children underwent lens extraction combined with anterior vitrectomy and SF-PCIOL under general anesthesia. The conjunctiva was opened, and 2 triangular and partial-thickness scleral flaps were created at the 2 and 8 o'clock positions. A scleral tunnel incision was then opened. This was either $3 \mathrm{~mm}$ for the foldable IOL (11 eyes) or $7 \mathrm{~mm}$ for the rigid polymethyl methacrylate (PMMA) IOL (36 eyes), through which the lens and the capsule bag were entirely removed with stable infusion in the anterior chamber. After lens extraction, a 23-gauge needle was placed in a traverse position through the scleral incision into the anterior chamber in order to perform a vitrectomy. All IOLs were fixated under scleral flaps with 9-0 and 10-0, 1-2 mm polypropylene suture posterior to the limbus. The IOL used in this study was either CZ70BD $(n=36)$ (Alcon Laboratories, Fort Worth, TX, USA), SA60AT $(n=9)$ (Alcon Laboratories, Fort Worth, TX, USA), or LI61SE $(n=2)$ (Bausch \& Lomb, St. Rochester, NY, USA).

UCVA, BCVA, refraction, IOP, slit-lamp examination, and direct ophthalmoscopy were examined regularly, and all complications were recorded at each visit. All data were analyzed by using SPSS (version 23.0; SPSS, Inc., Chicago, IL, USA). Comparison of visual acuity and refraction in the preoperative versus postoperative procedures and the PMMA versus foldable IOL was performed by using the Wilcoxon signed-rank test and the Mann-Whitney $\mathrm{U}$ test, respectively. Significance was defined by a $p$ value $<0.05$.

Intraocular Lens Implants in Children with Congenital Ectopia Lentis

\section{Results}

The average age of the patients at surgery was $61.6 \pm$ 22.3 months, and the median follow-up was $33.0 \pm 46.0$ months (range, 4-129). One patient was diagnosed with secondary glaucoma before surgery, which was caused by severe subluxated lens. After surgery, the IOP of this patient was normal.

\section{Visual and Refractive Outcomes}

The preoperative to postoperative changes in median $\operatorname{logMAR}$ UCVA and logMAR BCVA as well as the absolute values of spherical, astigmatism, and spherical equivalent are shown in Table 1. The UCVA and BCVA improved significantly after surgery $(p<0.001)$. In addition, the absolute values of spherical parameters decreased considerably $(p<0.001)$. The absolute values of astigmatism and the spherical equivalent showed no significant difference.

The comparisons between the foldable IOL and rigid PMMA IOL in the median logMAR UCVA and logMAR BCVA as well as the astigmatism and the spherical equivalent values are shown in Table 2. The astigmatism was lower in the foldable IOL compared with the rigid PMMA IOL ( $p=0.026)$, but neither VA nor BCVA showed any significant differences between the 2 types of IOL.

\section{Complications}

No intraoperative complications were found. All observed postoperative complications are described in Table 3. Pupillary capture was observed in 4 eyes, but this was resolved spontaneously in all the patients. IOL decentration occurred in 4 eyes, with the IOL haptics being broken in 3 eyes and suture degradation occurring in 1 eye. All eyes accepted the IOL replacements. More data with respect to these 4 eyes are shown in Table 4 . One eye had hypotony as well as showing choroid detachment, postoperatively. Although the IOP was normal after 5 days, 


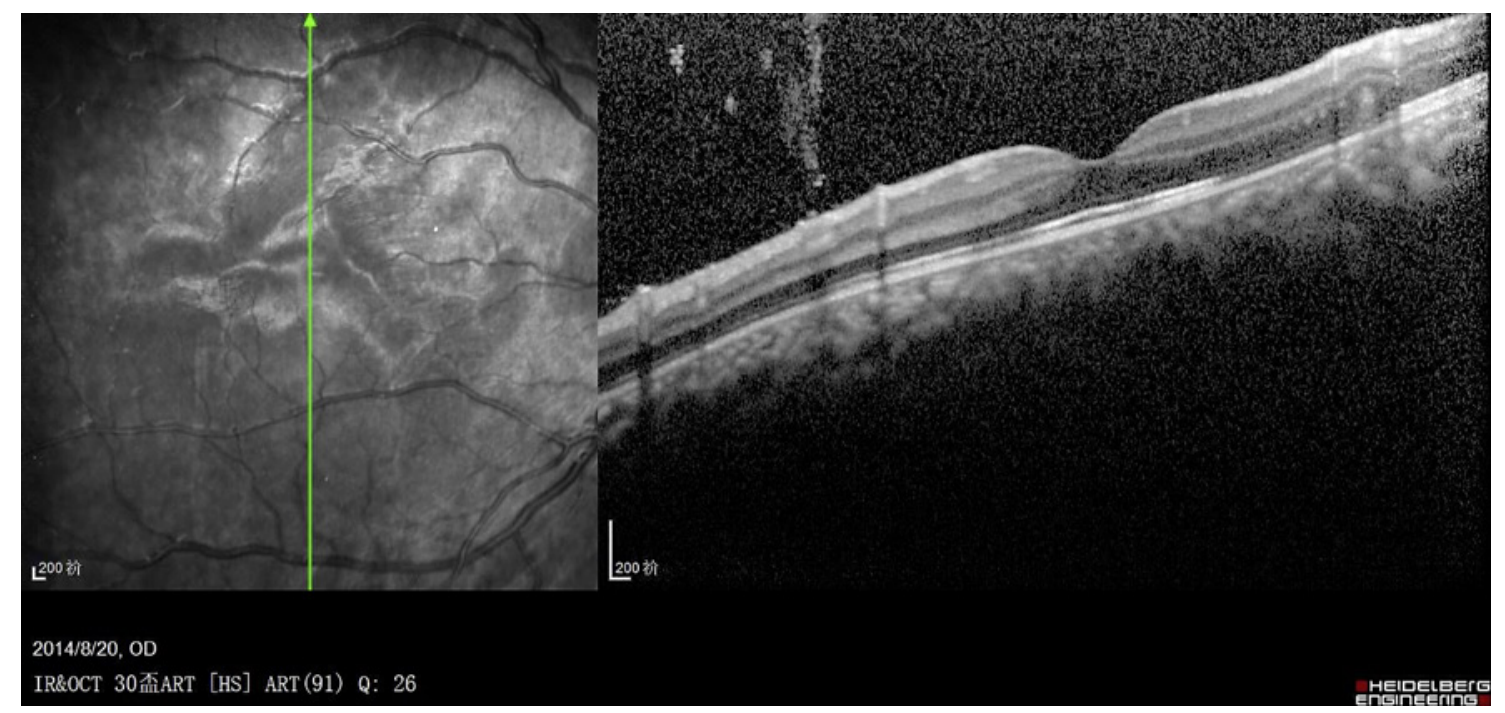

Fig. 1. One eye with a choroid edema which was observed using OCT at 1 week after surgery.

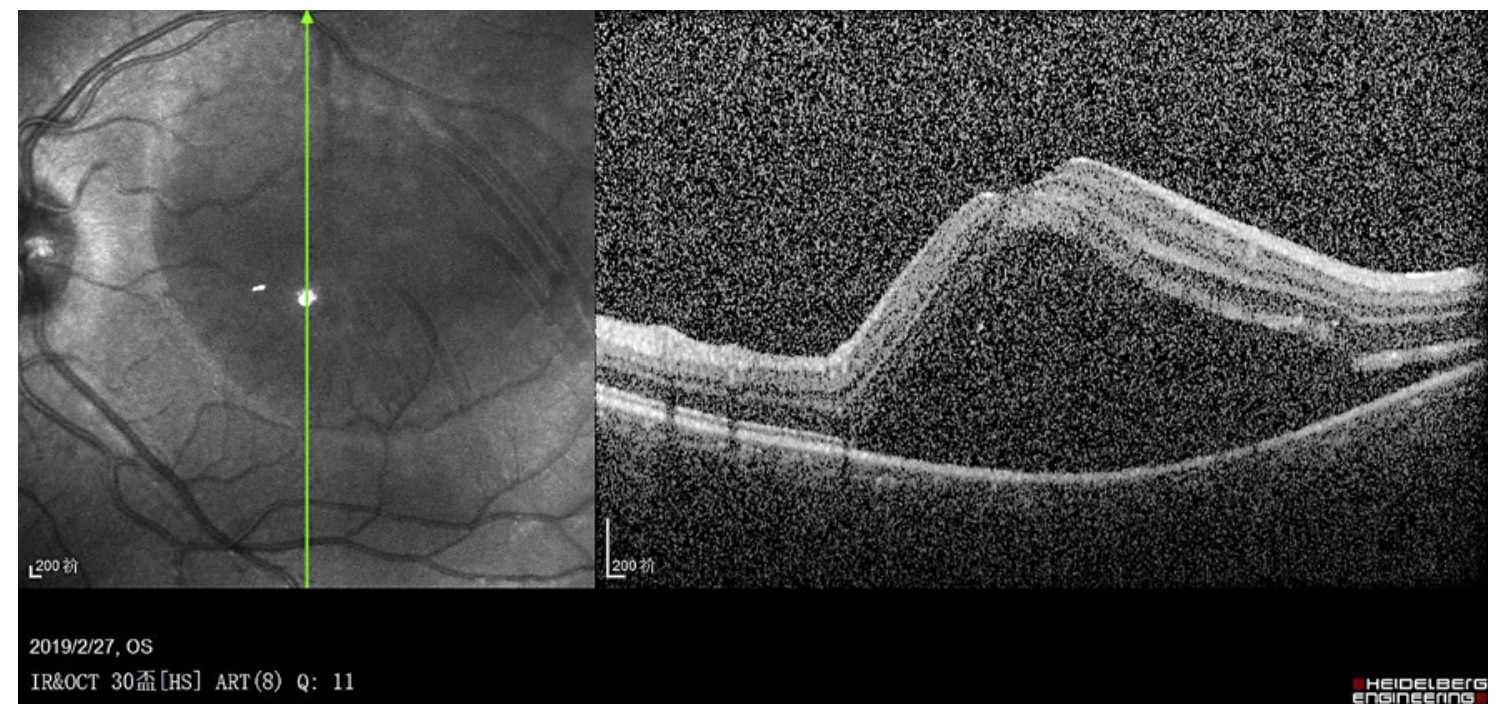

Fig. 2. A subretinal hemorrhage of 1 eye as shown using OCT, and this involved the superior temporary and the macular area with an overall area of approximately $4 \mathrm{PD}$.

choroid edema was still observed by optical coherence tomography until 1 month after surgery (Fig. 1). After conservative treatment, the BCVA reached logMAR 0. But, the IOL haptics was found to be broken in both eyes at the sixth year of follow-up. The IOLs were replaced, and the BCVA was logMAR 0 in both eyes, postoperatively. Subretinal hemorrhage was found at 1 week and after 2 months, postoperatively (Fig. 2, 3). After 2 months, all the hemorrhage was found to be absorbed, and the BCVA was logMAR 0.15.
The rate of secondary surgery which caused IOL decentration of the IOL haptics being broken and suture degradation was 9\% (Table 3). Three of the eyes with broken IOL haptics eventually underwent IOL replacement. A broken IOL haptics was found in 1 eye at 1 day, postoperatively, and this was found to be cut off because of being over tightened by 10-0 polypropylene suture (Fig. 4,5 ). In another patient, both eyes had broken IOL haptics of LI61SE type, simultaneously at 72 months. Suture degradation was also observed in one of the eyes of a single pa- 


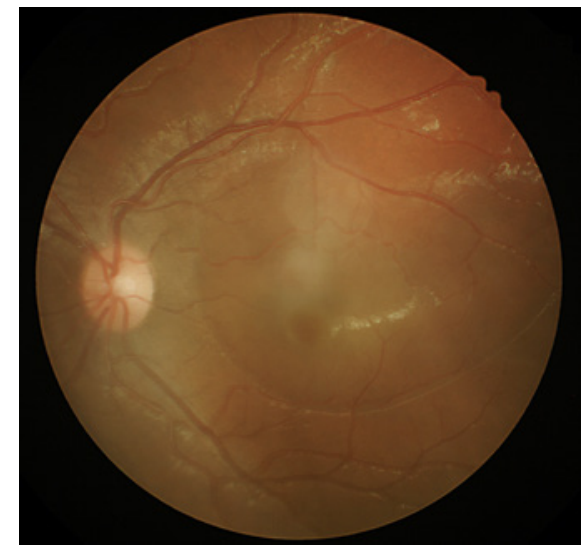

Fig. 3. One eye showing a subretinal hemorrhage in the fundus photographed at 1 week postoperatively.

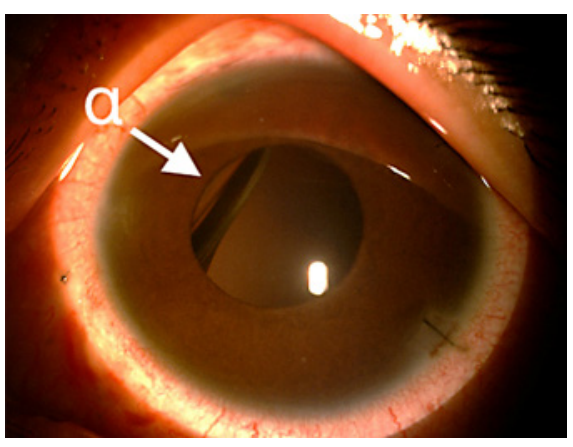

Fig. 4. One eye with IOL decentration on day 1 , postoperatively. The IOL haptic was seen through the pupil which is marked as a. IOL, intraocular lens.

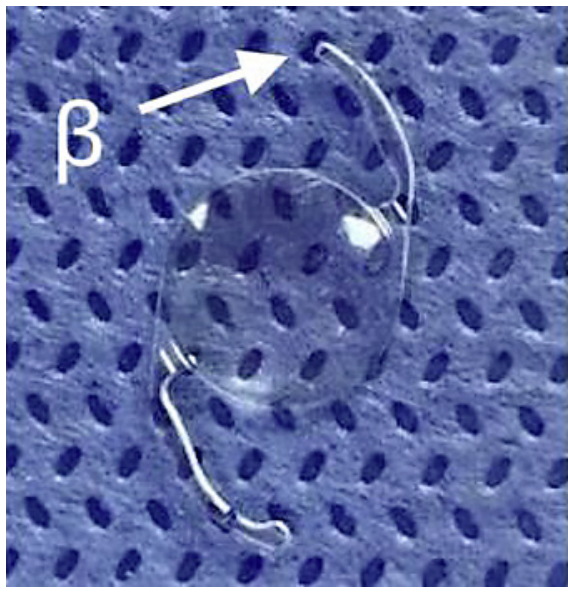

Fig. 5. Image of an example of broken IOL haptics of SA60AT type from 1 eye. Please note that the broken haptic is marked as $\beta$. IOL, intraocular lens. tient at 7 months after the procedure, and this was corrected by IOL re-fixation surgery. There were no cases of retinal detachment, endophthalmitis, or glaucoma as observed during the follow-ups of any of the patients in this study.

\section{Discussion}

The surgery which involved removal of the ectopia lentis and suturing a SF-PCIOL has been shown to be an effective technique which can improve the VA and BCVA in adults and children. Luebke et al. [15] reported that the BCVA was $0.1 \log$ MAR after sutured SF-PCIOL in adults with Marfan syndrome, with a follow-up of 48 months. However, the BCVA seemed to be lower in children, and these were mostly associated with severe amblyopia due to early ectopia of the lens. Sen et al. [13] described a BCVA of $0.35 \log$ MAR in children at $47.05 \pm 48.11$ months postoperatively. In our results, the median BCVA was $0.15 \log$ MAR after a 33.0-month follow-up. The better BCVA of our patients may be associated with the earlier surgical age which was $61.6 \pm 22.3$ months compared to $134.4 \pm 50.4$ months [13].

Targeted refraction depends on the age of children. In our study, surgery can considerably change the refraction experienced. Although astigmatism showed no significant difference after surgery, the diopter of the average spherical value was decreased by $>92 \%$. This is probably the main reason for the improvement seen in the VA and
BCVA. The median absolute value of spherical was 0.75 $\mathrm{D}$, which was acceptable for a child of about 5 years of age. Although the median astigmatism was higher in PMMA IOL $(2.50 \mathrm{D})$ when compared to foldable IOL (1.00 D; $p$ $=0.026)$, the VA, BCVA, and refraction parameters were similar in the 2 groups. Considering the limited data with respect to foldable IOL, a further study is needed to confirm these results.

Pupillary capture is a common complication of Marfan syndrome after SF-PCIOL. It has a reported rate of $20.5 \%$ in children with this condition [13]. The reasons may be that abnormal fibrillin metabolism occurs in Marfan syndrome, and this may lead to delays in the development of the ciliary body, which is rich in fibrillin, resulting in poor development of the iris sphincter and dilator muscles [16]. Furthermore, the IOL type used and the distance from the fixation point to the limbus as well as the absence of a vitreous body might also attribute to pupillary capture [17]. The incidence in our cases was relatively lower than others, which may be due to the shorter follow-up and longer distance between the limbus and the fixation point [13].

There were considerable rates of IOL decentration after PC-SFIOL in previous studies [11, 18]. However, with the development of surgical techniques, the rates have declined. In children with sutured SF-PCIOL, the incidence of IOL decentration varied from $4.6 \%$ to $24 \%$ [ 11 , $13,19]$. In the series with longer follow-up periods and younger age of patients, the IOL decentration seemed to occur more frequently $[11,18]$. The reason may be that 
children have a more active lifestyle and are perhaps less careful than adult populations. These reasons may accelerate the breakage of suture materials and produce a higher probability of eye trauma [12]. Polypropylene sutures were found to lack long survival times due to a slow degeneration which occurred over time, with a mean breakage time of 4 years [18]. In our patients, the incidence of IOL decentration was $9 \%$ (4 eyes) with 33.0-month follow-up, including broken IOL haptics in 3 eyes and suture degeneration in 1 eye. All the patients subsequently underwent secondary surgery. The relatively lower rate of IOL decentration may be associated with the younger age and shorter follow-up periods in our children. It is envisaged that with prolonged time, the rate will increase.

Choroid edema and hemorrhage were not rare complications after SF-PCIOL. In our patients, choroid edema was seen in $2 \%$ of children, which was secondary to postoperative transient hypotony, and this was readily treated with steroids. Subretinal hemorrhage occurred in 1 eye (2\%) at 1 week, postoperatively, and this was finally resolved spontaneously. We supposed that the hemorrhage was associated with the suturing, and this was tracked back to the macular area with a standing position. The rate of hemorrhage may be reduced by measures such as maintaining a pressurized globe, avoiding the use of 3 and 9 o'clock positions when creating the scleral flaps, minimizing the trans-scleral needle passes over the tissues, inserting the needles perpendicular to the ocular surface with an ab external technique, and avoiding extraocular blood wicking along the suture into the eye [12, $20,21]$. Severe complications that affect visual acuity, such as glaucoma, retinal detachment, and endophthalmitis, did not develop in our study.

In conclusion, although there are risks of secondary surgery, our data suggest that sutured SF-PCIOL is an effective technique for children under the age of 9 years with congenital ectopia lentis. Our study suggests that young children benefit from this type of clinical intervention. However, there is a need for more modern tech- niques to be proposed in order to improve the surgical outcome in these children and to reduce the incidence of postoperative complications.

\section{Acknowledgment}

The authors are grateful to Dr. Dev Sooranna of Imperial College London for English language edits of the manuscript.

\section{Statement of Ethics}

This study was performed in line with the principles of the Declaration of Helsinki. Approval was granted by the Ethics Committee of the First Affiliated Hospital of Guangxi Medical University (No. 2020.KY-E-171). All data were collected anonymously and retrospectively, and the requirement of written informed consent was therefore waived.

\section{Conflict of Interest Statement}

The authors have no conflicts of interest to declare.

\section{Funding Sources}

The authors have no funding source to declare.

\section{Author Contributions}

The study was designed by Xia Li and Xuyun Meng. Material preparation and data collection were performed by Xuyun Meng, Xi Cao, Yiyue Jia, and Jianhui Pan. Data analysis was performed by Xuyun Meng. The manuscript was written by Xuyun Meng and reviewed and edited by Xia Li and Yi Du. All authors read and approved the final manuscript.

\section{Data Availability Statement}

All data included in this study are available upon request by contact with the corresponding author.

\section{References}

1 Fuchs J, Rosenberg T. Congenital ectopia lentis. A Danish national survey. Acta Ophthalmol Scand. 1998;76(1):20-6.

2 Wagoner MD, Cox TA, Ariyasu RG, Jacobs DS, Karp CL. Intraocular lens implantation in the absence of capsular support: a report by the American Academy of Ophthalmology. Ophthalmology. 2003;110(4):840-59.
3 Yen KG, Reddy AK, Weikert MP, Song Y, Hamill MB. Iris-fixated posterior chamber intraocular lenses in children. Am J Ophthalmol. 2009 Jan;147(1):121-6.

4 Gawdat GI, Taher SG, Salama MM, Ali AA. Evaluation of Artisan aphakic intraocular lens in cases of pediatric aphakia with insufficient capsular support. J AAPOS. 2015 Jun;19(3): 242-6. 
5 Shah R, Weikert MP, Grannis C, Hamill MB, Kong L, Yen KG. Long-term outcomes of irissutured posterior chamber intraocular lenses in children. Am J Ophthalmol. 2015 Jan;161: 44-9.e1.

6 Faria MY, Ferreira N, Neto E. Retropupillary iris-claw intraocular lens in ectopia lentis in Marfan syndrome. Int Med Case Rep J. 2016; 9:149-53.

7 Catala-Mora J, Cuadras D, Diaz-Cascajosa J, Castany-Aregall M, Prat-Bartomeu J, GarciaArumi J. Anterior iris-claw intraocular lens implantation for the management of nontraumatic ectopia lentis: long-term outcomes in a paediatric cohort. Acta Ophthalmol. 2017 Mar;95(2):170-4.

8 Dietlein TS, Jacobi PC, Konen W, Krieglstein GK. Complications of endocapsular tension ring implantation in a child with Marfan's syndrome. J Cataract Refract Surg. 2000;26: 937-40.

9 Byrd JM, Young MP, Liu W, Zhang Y, Tate DB, Crandall AS, et al. Long-term outcomes for pediatric patients having transscleral fixation of the capsular bag with intraocular lens for ectopia lentis. J Cataract Refract Surg. 2018 May;44(5):603-9.
10 Bardorf CM, Epley KD, Lueder GT, Tychsen L. Pediatric transscleral sutured intraocular lenses: efficacy and safety in 43 eyes followed an average of 3 years. J AAPOS. 2004;8(4): $318-24$.

11 Asadi R, Kheirkhah A. Long-term results of scleral fixation of posterior chamber intraocular lenses in children. Ophthalmology. 2008; 115(1):67-72.

12 Buckley EG. Safety of transscleral-sutured intraocular lenses in children. J AAPOS. 2008; 12(5):431-9.

13 Sen P, Attiku Y, Bhende P, Rishi E, Ratra D, Sreelakshmi K. Outcome of sutured scleral fixated intraocular lens in Marfan syndrome in pediatric eyes. Int Ophthalmol. 2020 Jun; 40(6):1531-8.

14 Medsinge A, Nischal KK. Pediatric cataract: challenges and future directions. Clin Ophthalmol. 2015;9:77-90.

15 Luebke J, Reinhard T, Agostini H, Boehringer D, Eberwein P. Long-term follow-up after scleral lens fixation in patients with Marfan syndrome. BMC Ophthalmol. 2017 Dec 6; 17(1):235.

16 Wheatley HM, Traboulsi EI, Flowers BE, Maumenee IH, Azar D, Pyeritz RE, et al. Immunohistochemical localization of fibrillin in human ocular tissues. Relevance to the Marfan syndrome. Arch Ophthalmol. 1995; 113(1):103-9.
17 Cho BJ, Yu HG. Surgical outcomes according to vitreous management after scleral fixation of posterior chamber intraocular lenses. Retina. 2014;34(10):1977-84.

18 Vote BJ, Tranos P, Bunce C, Charteris DG, Da Cruz L. Long-term outcome of combined pars plana vitrectomy and scleral fixated sutured posterior chamber intraocular lens implantation. Am J Ophthalmol. 2006 Feb;141(2):30812.

19 Sen P, Vinay Kumar S, Bhende P, Rishi P, Rishi E, Rao C, et al. Surgical outcomes and complications of sutured scleral fixated intraocular lenses in pediatric eyes. Can J Ophthalmol. 2017 Feb;53(1):49-55.

20 Soong HK, Meyer RF, Sugar A. Techniques of posterior chamber lens implantation without capsular support during penetrating keratoplasty: a review. Refract Corneal Surg. 1989; 5(4):249-55.

21 Lane SS, Lubniewski AJ, Holland EJ. Transsclerally sutured posterior chamber lenses: improved lens designs and techniques to maximize lens stability and minimize suture erosion. Semin Ophthalmol. 1992 Dec;7(4): $245-52$. 